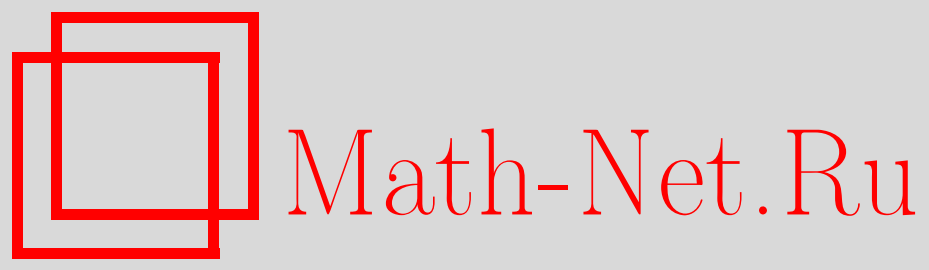

Н. П. Долбилин, Ю. М. Зиновьев, А. С. Мищенко, М. А. Штанько, М. И. Штогрин, Гомологические свойства димерных покрытий решеток на поверхностях, Функи. анализ и его прил., 1996, том 30, выпуск 3, 19-33

DOI: https://doi.org/10.4213/faa535

Использование Общероссийского математического портала Math$\mathrm{Net.Ru}$ подразумевает, что вы прочитали и согласны с пользовательским соглашением http://www . mathnet.ru/rus/agreement

Параметры загрузки:

IP : 54.147 .182 .235

26 апреля 2023 г., 16:59:12

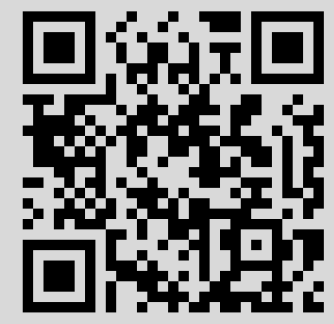


Функииональньй анализ и еәо приложения

1996, т. 30, вып. 3, с. 19-33

УДК $514.8+515.162$

\section{Гомологические свойства димерных покрытий решеток на поверхностях}

(c) 1996. Н. П. ДолБИлИн, Ю. М. ЗиновьЕВ, А. С. МИЩЕнко, М. А. Штанько, М. И. ШтогРИн ${ }^{1}$

\section{$\S 1$. Введение}

В литературе известно весьма ограниченное число примеров, в которых статистическая сумма для модели Изинга вычисляется точно. При вычислении статистической суммы двумерной модели Изинга математически наиболее законченным является вывод, основанный на геометрических соображениях (см., например, [1]). Этот элегантный вывод использует представление статистической суммы в виде линейной комбинации пфаффианов структурных матриц решетки, естественно связанных с функцией взаимодействия и ориентациями. Однако это представление существенно зависит от рассматриваемых граничных условий на решетке. В случае свободных условий и в случае периодических условий в одном измерении статистическая сумма выражается через один пфаффиан. В случае же двояко-периодических условий необходимо использовать пфаффианы четырех структурных матриц.

Целью настоящей работы является выяснение геометрической природы зависимости представлений статистической суммы модели Изинга в виде линейной комбинации пфаффианов структурных матриц от выбора граничных условий. Оказалось, что эта зависимость имеет чисто гомологический смысл. Каждая решетка может быть расположена на поверхности. Так, решетка двумерной модели Изинга со свободными граничными условиями расположена на плоскости для граничных условий, периодических в одном измерении, она расположена на цилиндрической поверхности, а для двояко-периодических граничных условий решетка расположена на торе.

Выражение статистической суммы через пфаффианы структурных матриц зависит от гомологических свойств той поверхности, на которой расположена решетка. При этом комбинаторная структура решетки может быть совершенно произвольной.

В $§ 2$ настоящей работы приводится представление статистической суммы в виде производящей функции димерных покрытий на расширенном графе, который расположен на той же поверхности, и приводятся все необходимые обозначения и формулы. В $\S \S 3,4$ описываются гомологические препятствия к выражению производящей функции через пфаффианы структурных матриц и выводятся основные формулы.

\footnotetext{
${ }^{1}$ Работа выполнена при поддержке Российского фонда фундаментальных исследований (грант 96-01-00167) и International Science Foundation.
} 


\section{§2. Статистическая сумма модели Изинга и димерные покрытия}

Рассмотрим конечный граф Г . В качестве характерного примера такого графа будем рассматривать прямоугольную решетку на плоскости, составленную из точек с целыми декартовыми координатами $x=j, y=k, 0 \leqslant j \leqslant N$, $0 \leqslant k \leqslant M$, и соответствуюших горизонтальных и вертикальных отрезков, причем противоположные стороны всего прямоугольника отождествлены. Другими словами, рассматривается прямоугольная решетка на торе. Вершины графа $\Gamma$ будем обозначать буквами $p, q, \ldots$, а ребра - буквами $\alpha, \beta, \ldots$ Множество всех вершин, т. е. нульмерный остов, будем обозначать через $V$, а множество всех ребер - через $L$. Каждое ребро $\alpha$ графа соединяет две вершины $(p, q)$. Предположим, что $p \neq q$ и каждое ребро определяется своими концами единственным образом. Тогда будем писать $\alpha=(p, q)$. Фиксируем некоторое разбиение множества ребер $L$ на конечное число подмножеств $L_{i}$ :

$$
L=\bigcup_{i=1}^{r} L_{i}, \quad L_{i} \cap L_{j}=\varnothing, \quad i \neq j .
$$

Пусть на вершинах графа $\Gamma$ задана функция $\sigma$ со значениями в группе $\mathbb{Z}_{2}=$ $\{+1,-1\}$. Энергия взаимодействия $H(\sigma)$ модели Изинга с нулевым магнитным полем может быть представлена в виде

$$
H(\sigma)=-\sum_{i=1}^{r} E_{i} \sum_{\alpha \in L_{i}} \partial^{*} \sigma(\alpha)
$$

где $\partial^{*} \sigma((p, q))=\sigma(p) \sigma(q)$ и $E_{i}$ - значение энергии, соответствующей ребрам подмножества $L_{i}$. Статистическая сумма модели Изинга с нулевым магнитным полем определяется следующим образом:

$$
Z_{\Gamma}=\sum_{\sigma \in C^{0}\left(\Gamma, \mathbb{Z}_{2}\right)} \exp \{-\beta H(\sigma)\},
$$

где через $C^{0}\left(\Gamma, \mathbb{Z}_{2}\right)$ мы обозначили группу функций на вершинах графа $\Gamma$ со значениями в группе $\mathbb{Z}_{2}$. Производя преобразование Фурье на группе $C^{0}\left(\Gamma, \mathbb{Z}_{2}\right)$ и используя стандартные теоремы гармонического анализа либо пользуясь доказательством, предложенным в работах [2] и [1, гл. 5], выражение $(2.3)$ можно преобразовать к виду

$$
Z_{\Gamma}=2^{\#(V)} \prod_{i=1}^{r}\left(\operatorname{ch} \beta E_{i}\right)^{\#\left(L_{i}\right)} \sum_{\xi \in Z_{1}\left(\Gamma, \mathbb{Z}_{2}\right)} \prod_{i=1}^{r}\left(\operatorname{th} \beta E_{i}\right)^{k_{i}(\xi)},
$$

где через \#(V) обозначено число вершин графа $\Gamma$, через \# $\left(L_{i}\right)$ - число ребер из множества $L_{i}$, а через $k_{i}(\xi)$ - число ребер из множества $L_{i}$, на которых цикл $\xi \in Z_{1}\left(\Gamma, \mathbb{Z}_{2}\right)$ принимает значение -1. Группа циклов $Z_{1}\left(\Gamma, \mathbb{Z}_{2}\right)$ определяется как группа функций на ребрах графа $\Gamma$ со значениями в группе $\mathbb{Z}_{2}$, причем для любой вершины графа $\Gamma$ произведение значений функции на ребрах, соединяющих эту вершину с другими вершинами, равно 1.

Введем новый объект на графе Г. Димерным покрытием будем называть систему ребер $U=\left\{\alpha_{k}=\left(p_{k}, q_{k}\right)\right\}$, удовлетворяющую следуюшим условиям: 
(1) ребра димерного покрытия попарно не пересекаются, т. е.

$$
\partial \alpha_{j} \cap \partial \alpha_{k}=\varnothing, \quad j \neq k
$$

(2) все вершины покрываются ребрами димерного покрытия, т. е.

$$
\bigcup_{\alpha_{k} \in U} \partial \alpha_{k}=V .
$$

Следуя идеям работы [3], мы установим взаимно однозначное соответствие между элементами группы циклов $Z_{1}\left(\Gamma, \mathbb{Z}_{2}\right)$ и димерными покрытиями некоторого другого расширенного графа. Для простоты мы будем предполагать, что каждая вершина исходного графа $Г$ соединена с четным числом (большим двух) других вершин. Пусть вершина $p \in \Gamma$ соединена ребрами $\mathrm{c} 2 k, k>1$, другими вершинами. Тогда в новом графе заменим вершину $p$ на $2 k-2$ вершин и добавим к старым $2 k$ ребрам еще $2 k-3$ ребер, соединяющих новые вершины. При этом к первой и последней вершинам подходят два старых ребра и одно новое, а к остальным вершинам подходят одно старое и два новых ребра. Таким образом, каждая вершина соединена ровно с тремя другими вершинами. Проводя это построение для каждой вершины графа $\Gamma$, получим новый граф $\operatorname{tr}(\Gamma)$. Если на ребрах графа $\Gamma$ была задана функция $\xi \in Z_{1}\left(\Gamma, \mathbb{Z}_{2}\right)$, то она также задана на старых ребрах графа $\operatorname{tr}(\Gamma)$. Продолжим эту функцию до $\mathbb{Z}_{2}$-значной функции $\hat{\xi}$ на ребрах графа $\operatorname{tr}(\Gamma)$ с помощью условия $\partial \hat{\xi}=-1$, означающего, что в каждой вершине графа $\operatorname{tr}(\Gamma)$ произведение значений функции $\hat{\xi}$ на ребрах, соединяющих эту вершину с тремя другими вершинами графа $\operatorname{tr}(\Gamma)$, равно -1 . Поскольку функция $\xi$ является циклом, т. е. принадлежит группе $Z_{1}\left(\Gamma, \mathbb{Z}_{2}\right)$, и каждой вершине графа $\Gamma$ соответствует четное число вершин графа $\operatorname{tr}(\Gamma)$, то продолжение $\hat{\xi}$ на ребра графа $\operatorname{tr}(\Gamma)$ определено однозначно. Обратно, каждая функция $\hat{\xi} \in C^{1}\left(\operatorname{tr}(\Gamma), \mathbb{Z}_{2}\right)$, удовлетворяющая условию $\partial \hat{\xi}=-1$, при сужении на ребра графа $\Gamma$ определяет цикл из группы $Z_{1}\left(\Gamma, \mathbb{Z}_{2}\right)$.

Каждую вершину графа $\operatorname{tr}(\Gamma)$ заменим на треугольник, из каждой вершины которого исходит одно ребро графа $\operatorname{tr}(\Gamma)$ и два ребра треугольника. Полученный граф обозначим через $\operatorname{cl}(\Gamma)$. Множество функщий $\hat{\xi} \in C^{1}\left(\operatorname{tr}(\Gamma), \mathbb{Z}_{2}\right)$, удовлетворяющих условию $\partial \hat{\xi}=-1$, находится во взаимно однозначном соответствии с множеством димерных покрытий графа $\mathrm{cl}(\Gamma)$. В самом деле, включим в димерное покрытие $U(\hat{\xi})$ все ребра, на которых функция $\hat{\xi}$ принимает значение -1 . Тогда к каждому треугольнику подходят либо три таких ребра, либо одно. В первом случае все вершины треугольника покрыты и ни одно ребро треугольника не принадлежит димерному покрытию $U(\hat{\xi})$. Во втором случае в димерное покрытие $U(\hat{\xi})$ входит сторона треугольника, противоположная вершине, к которой подходит ребро димерного покрытия $U(\hat{\xi})$. Тогда опять все вершины треугольника покрыты ребрами из $U(\hat{\xi})$ и ребра из $U(\hat{\xi})$ не пересекаются между собой.

Пусть дано димерное покрытие $U$ графа $\operatorname{cl}(\Gamma)$. Построим функцию $\hat{\xi}(U)$, полагая ее равной 1 на ребрах графа $\operatorname{tr}(\Gamma)$, не входящих в димерное покрытие $U$, и -1 на ребрах графа $\operatorname{tr}(\Gamma)$, входящих в димерное покрытие $U$. Если сторона треугольника входит в димерное покрытие $U$, то к треугольнику подходит одно 
ребро этого димерного покрытия. Если же стороны треугольника не входят в димерное покрытие $U$, то к треугольнику подходят три ребра этого димерного покрытия. Таким образом, функция $\hat{\xi}(U)$ удовлетворяет условию $\partial \hat{\xi}(U)=-1$.

Доказанное соответствие позволяет переписать статистическую сумму (2.4) в другом виде. Каждому димерному покрытию $U$ графа $\mathrm{cl}(\Gamma)$ взаимно однозначно соответствует цикл $\xi \in Z_{1}\left(\Gamma, \mathbb{Z}_{2}\right)$, причем ребро $\alpha$ принадлежит $U \cap \Gamma$ тогда и только тогда, когда $\xi(\alpha)=-1$. Таким образом соотношение (2.4) приобретает вид

$$
Z_{\Gamma}=2^{\#(V)} \prod_{i=1}^{r}\left(\operatorname{ch} \beta E_{i}\right)^{\#\left(L_{i}\right)} \sum_{U} \prod_{i=1}^{r}\left(\operatorname{th} \beta E_{i}\right)^{\#\left(U \cap L_{i}\right)},
$$

где суммирование идет по всем димерным покрытиям $U$ графа $\operatorname{cl}(\Gamma)$, а \# $\left(U \cap L_{i}\right)$ означает число ребер из димерного покрытия $U$, входящих в множество $L_{i}$.

ОПРЕДЕЛЕНИЕ. Производящей функиией димерных покрытий графа Г разбиением (2.1) будем называть функцию

$$
Z=\sum_{U} z_{1}^{\#\left(U \cap L_{1}\right)} \cdots z_{r}^{\#\left(U \cap L_{r}\right)}=\sum_{U} z^{U}
$$

где $z^{U}=\prod_{i} z_{i}^{\#\left(U \cap L_{i}\right)}$ и сумма берется по всем димерным покрытиям графа $\Gamma$.

Совершенно ясно, что, для того чтобы существовало хотя бы одно димерное покрытие, необходимо, чтобы число вершин $M=\#(V)$ было четным.

Если $\alpha \in L_{i}$, то скажем, что $i=i(\alpha)$. Пусть на графе $\Gamma$ выбрано направление каждого ребра $\alpha \in L$. Если $\alpha$ определяется своими концами $(p, q)$, то направление ребер можно задать в виде функции $f(p, q)$, принимающей только два значения \pm 1, причем

$$
f(p, q)=-f(q, p) .
$$

Тогда началом ребра $\alpha=(p, q)$ будет точка $p$, а концом - точка $q$, если $f(p, q)=1$.

Итак, пусть функция (2.9) задана. Определим кососимметрическую матрицу $A=\left\|a_{p, q}\right\|$, размер которой равен числу $M$ вершин графа $Г$. Элементы $a_{p, q}$ матрицы $A$ нумеруются вершинами графа Г. Положим

$$
a_{p, q}= \begin{cases}0, & \text { если } p, q \text { не соединяются ребром, } \\ f(p, q) z_{i(p, q)}, & \text { если } p, q \text { соединяются ребром. }\end{cases}
$$

Определение (2.10) пока не задает матрищу, поскольку необходимо еще задать некоторое линейное упорядочение вершин, которыми индексируются элементы матрицы $A$. Таким образом, определение матрицы $A$ зависит от выбора функции $f$ и дополнительно от линейного упорядочения вершин. Фиксируем некоторое линейное упорядочение вершин графа $\Gamma$.

Очевидно, что матрица $A$ кососимметрична. Поэтому мы можем определить ее пфаффиан по формуле

$$
\operatorname{Pf}(A)=((M / 2) !)^{-1} 2^{-M / 2} \sum_{\pi}(-1)^{\sigma(\pi)} a_{p_{1}, p_{2}} \cdots a_{p_{(M-1)}, p_{M}},
$$


где $\pi=\left(p_{1}, \ldots, p_{M}\right)$ - произвольная перестановка, а $\sigma(\pi)$ - знак этой перестановки (относительно линейного упорядочения вершин в множестве $V$ ). Наше определение пфаффиана отличается от стандартного определения $[4$, гл. $9, \S 5.2]$ лишь множителем $((M / 2) !)^{-1}$.

Пфаффиан $\operatorname{Pf}(A)$ матрицы $A$ зависит определенным образом от выбора функции $f$ и упорядочения вершин. Если поменять упорядочение вершин на другое, то в формуле (2.11) изменится знак в случае, если перестановка, меняющая упорядочение, нечетна.

Пусть $U=\left\{\alpha_{k}=\left(p_{k}, q_{k}\right)\right\}$ - димерное покрытие. Через $\sigma(U)$ обозначим четность перестановки $\left(p_{1}, q_{1}, p_{2}, q_{2}, \ldots, p_{M / 2}, q_{M / 2}\right)$, которая, очевидно, не зависит от выбора упорядочения ребер в димерном покрытии $U$. Однако четность этой перестановки зависит от упорядочения вершин отдельного ребра. Тем не менее моном $(-1)^{\sigma(U)} \prod_{k} a_{p_{k}, q_{k}}$ не меняется ни при изменении упорядочения ребер в димерном покрытии, ни при изменении порядка вершин каждого отдельного ребра.

ТЕорема 1. Пфаффиан может быть выражен формулой

$$
\operatorname{Pf}(A)=\sum_{U}(-1)^{\sigma(U)} \prod_{k} a_{p_{k}, q_{k}},
$$

где сумма берется по всем димерным покрытиям.

Таким образом, мы получаем следующую формулу:

$$
\begin{aligned}
\operatorname{Pf}(A) & =\sum_{U}(-1)^{\sigma(U)} \prod_{k} f\left(p_{k}, q_{k}\right) z_{i\left(p_{k}, q_{k}\right)} \\
& =\sum_{U}(-1)^{\sigma(U)} \prod_{k} f\left(p_{k}, q_{k}\right) \prod_{i}\left(z_{i}\right)^{\#\left(U \cap L_{i}\right)} .
\end{aligned}
$$

Пфаффиан может быть выражен следующим образом:

$$
\operatorname{Pf}(A)=\sum_{U}(-1)^{\sigma(U)} \prod_{k} f\left(p_{k}, q_{k}\right) z^{U} .
$$

Здесь, как и ранее, число

$$
g(U)=(-1)^{\sigma(U)} \prod_{k} f\left(p_{k}, q_{k}\right)
$$

определяется независимо от представления димерного покрытия $U$ в виде последовательности (направленных) ребер

$$
U=\left\{\alpha_{k}=\left(p_{k}, q_{k}\right), 1 \leqslant k \leqslant M / 2\right\} .
$$

Число (2.15) можно также считать равным $(-1)^{\sigma(U)}$ для такого представления димерного покрытия $U$, в котором направления ребер совпадают с направлениями, задаваемыми функцией $f(p, q)$.

Итак, окончательное выражение имеет следующий вид:

$$
\operatorname{Pf}(A)=\sum_{U} g(U) z^{U}
$$




\section{§3. Производящая функция и пфаффиан}

Таким образом, для выражения производящей функции (2.8) через пфафффиан (2.16) желательно было бы найти такую функцию $f(p, q)$, чтобы

$$
g(U) \equiv \text { Const }
$$

для любого димерного покрытия $U$.

Пусть $U_{1}$ и $U_{2}$ - два димерных покрытия. Тогда условие (3.1) означает, что

$$
g\left(U_{1}\right)=g\left(U_{2}\right) \quad \text { или } \quad g\left(U_{1}\right) g\left(U_{2}\right)=1 .
$$

Рассмотрим симметрическую разность

$$
U_{1} \Delta U_{2}=\left(U_{1} \backslash U_{2}\right) \cup\left(U_{2} \backslash U_{1}\right) .
$$

Из определения димерного покрытия следует, что это множество представляется в виде несвязного объединения конечного числа замкнутых ломаных,

$$
U_{1} \Delta U_{2}=\bigcup_{i=1}^{s} \gamma_{i}
$$

причем ни одна ломаная $\gamma_{i}$ не имеет самопересечений, а ребра из $U_{1}$ и $U_{2}$ попеременно чередуются.

Функцию $g(U)$ удобно представлять в аддитивной форме:

$$
g(U)=(-1)^{\tau(U)},
$$

где $\tau(U)$ определяется следующим образом: пусть

$$
U=\left\{\left(p_{1}, q_{1}\right), \ldots,\left(p_{M / 2}, q_{M / 2}\right)\right\}
$$

- некоторое упорядочение ребер димерного покрытия. Тогда

$$
\tau(U)=\sigma\left(p_{1}, q_{1}, \ldots, p_{M / 2}, q_{M / 2}\right)+\sum_{i=1}^{M / 2} \varphi\left(p_{i}, q_{i}\right) \bmod 2,
$$

где

$$
f(p, q)=(-1)^{\varphi(p, q)} .
$$

Функция $\varphi(p, q)$ удовлетворяет следующему условию косой симметрии:

$$
\varphi(p, q)+\varphi(q, p)=1 \bmod 2 .
$$

Всякая другая функция $\varphi_{1}(p, q)$, удовлетворяющая условию (3.8), может быть выражена через функцию $\varphi(p, q)$ путем прибавления некоторой одномерной коцепи $x(p, q)$ со значениями в группе вычетов по $\bmod 2$, т. е. такой функции $x(p, q) \in \mathbb{Z}_{2}$ (здесь и далее мы тем же символом $\mathbb{Z}_{2}$ обозначаем группу вычетов по модулю 2), которая удовлетворяет условию

$$
x(p, q)+x(q, p)=0 \bmod 2 .
$$

А именно,

$$
\varphi_{1}(p, q)=\varphi(q, p)+x(p, q) \bmod 2 .
$$


Обратно, для любой коцепи (3.9) функция типа (3.10) удовлетворяет условию (3.8).

Условие (3.1) эквивалентно следующему тождеству:

$$
\tau(U) \equiv \text { Const } \bmod 2 .
$$

Для двух димерных покрытий $U_{1}$ и $U_{2}$ условие (3.2) можно записать в виде

$$
\tau\left(U_{1}\right)=\tau\left(U_{2}\right) \bmod 2 \text { или } \tau\left(U_{1}\right)+\tau\left(U_{2}\right)=0 \bmod 2 .
$$

Применим эти обозначения к случаю двух димерных покрытий. В соответствии с (3.4) выберем упорядочение ребер первого димерного покрытия $U_{1}$ таким образом, чтобы сначала шли ребра из кривой $\gamma_{1}$, потом из кривой $\gamma_{2}$ и т.д., вплоть до последней кривой $\gamma_{s}$; далее пусть идут ребра, не принадлежащие $U_{1} \Delta U_{2}$ :

$$
\begin{aligned}
U_{1}=\{ & \left(p_{1}, q_{1}\right), \ldots,\left(p_{l_{1}}, q_{l_{1}}\right) ; \\
& \left(p_{l_{1}+1}, q_{l_{1}+1}\right), \ldots,\left(p_{l_{1}+l_{2}}, q_{l_{1}+l_{2}}\right) ; \\
& \ldots \\
& \left(p_{l_{1}+\cdots+l_{s-1}+1}, q_{l_{1}+\cdots+l_{s-1}+1}\right), \ldots,\left(p_{l_{1}+\cdots+l_{s}}, q_{l_{1}+\cdots+l_{s}}\right) ; \\
& \left.\left(p_{l_{1}+\cdots+l_{s}+1}, q_{l_{1}+\cdots+l_{s}+1}\right), \ldots,\left(p_{M / 2}, q_{M / 2}\right)\right\} .
\end{aligned}
$$

у димерного покрытия $U_{2}$ последняя группа ребер та же самая. Первые же $s$ групп ребер покрытия $U_{2}$ отличаются от ребер покрытия $U_{1}$, но ребра покрытия $U_{2}$ покрывают ту же группу вершин, однако со сдвигом:

$$
\begin{aligned}
U_{2}=\{ & \left(q_{1}, p_{2}\right), \ldots,\left(q_{l_{1}}, p_{1}\right) ; \\
& \left(q_{l_{1}+1}, p_{l_{1}+2}\right), \ldots,\left(q_{l_{1}+l_{2}}, p_{l_{1}+1}\right) ; \\
& \ldots \\
& \left(q_{l_{1}+\cdots+l_{s-1}+1}, p_{l_{1}+\cdots+l_{s-1}+2}\right), \ldots,\left(q_{l_{1}+\cdots+l_{s}}, p_{l_{1}+\cdots+l_{s-1}+1}\right) ; \\
& \left.\left(p_{l_{1}+\cdots+l_{s}+1}, q_{l_{1}+\cdots+l_{s}+1}\right), \ldots,\left(p_{M / 2}, q_{M / 2}\right)\right\} .
\end{aligned}
$$

Теперь мы подсчитаем сумму $\tau\left(U_{1}\right)+\tau\left(U_{2}\right)$. Пользуясь формулой $(3.6)$, подсчитаем первое слагаемое, которое равно четности перестановки, переводящей (3.13) в (3.14). Эта перестановка, очевидно, состоит из циклических перестановок каждой группы вершин. Поскольку каждая группа вершин состоит из четного числа вершин, каждая циклическая перестановка нечетна. Следовательно, первое слагаемое равно числу групп вершин, т. е. $s$.

Второе слагаемое, определяемое функцией $\varphi(p, q)$, равно сумме значений Функции $\varphi(p, q)$, взятой по всем ребрам обоих покрытий $U_{1}$ и $U_{2}$. Те ребра, которые являются общими для обоих покрытий, дают нулевой вклад в сумму. Остальные же ребра организованы в конечное число замкнутых ломаных без самопересечений $\gamma_{i}, 1 \leqslant i \leqslant s$, каждая из которых обходится в некотором направлении. Поэтому вклад каждой замкнутой ломаной $\gamma_{i}$ равен $\varphi\left(\gamma_{i}\right)$ - сумме значений функции $\varphi(p, q)$, взятой по всем ребрам ломаной $\gamma_{i}$. При этом, как легко видеть, эта сумма не зависит от выбора направления, выбранного на ломаной $\gamma_{i}$, поскольку число ребер у ломаной $\gamma_{i}$ четно. 
Таким образом, имеет место следующая теорема:

Теорема 2. Пусть $U_{1}$ и $U_{2}$ - два димерных покрытия $u\left\{\gamma_{i}, i=1, \ldots, s\right\}$ - система попарно не пересекаюшихся замкнутых ломаных без самопересечения из формуль (3.4). Тогда

$$
\tau\left(U_{1}\right)+\tau\left(U_{2}\right)=\psi\left(U_{1} \Delta U_{2}\right)=\psi\left(\bigcup \gamma_{i}\right) \bmod 2,
$$

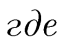

$$
\psi\left(U_{1} \Delta U_{2}\right)=\psi\left(\bigcup \gamma_{i}\right)=s+\sum_{i=1}^{s} \varphi\left(\gamma_{i}\right) \bmod 2
$$

\section{§4. Гомологические препятствия}

Мы попытаемся изучить препятствие к построению функции $\varphi(p, q)$ на графе $\Gamma$, удовлетворяющей условиям, которые позволяют выразить производящую функцию $Z$ через пфаффиан матрицы $A$. Это препятствие, как было показано в $\S 3$, состоит в том, чтобы функция $\psi(\gamma)$ тождественно равнялась нулю на всех замкнутых (вообще говоря, несвязных) ломаных без самопересечений, реализующих разность двух димерных покрытий.

Такие ломаные, разумеется, не исчерпывают любые замкнутые ломаные, даже без самопересечений, не говоря уж о произвольных замкнутых параметризованных ломаных. Описать класс ломаных, реализующих разность двух димерных покрытий, с помощью внутренних характеристик этих ломаных затруднительно. Однако они обладают некоторыми свойствами, которые являются, по крайней мере, необходимыми условиями для того, чтобы данная ломаная могла реализовывать разность двух димерных покрытий. Во-первых, число ребер у такой ломаной должно быть четным, поскольку ее ребра поочередно должны принадлежать двум димерным покрытиям. Значит, число вершин такой ломаной тоже должно быть четным. Во-вторых, число вершин, не покрытых данной ломаной, тоже должно быть четным, поскольку эти вершины должны быть покрыты ребрами из общей части двух димерных покрытий. Более того, ломаная разбивает весь граф Г на несколько компонент связности, так что в каждой компоненте связности число вершин должно быть четным.

Ниже мы везде будем предполагать, что

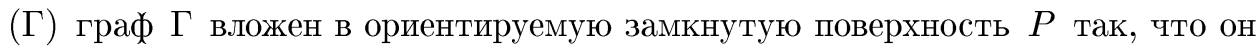
является одномерным остовом некоторого клеточного разбиения $C W$-комплекса.

Это клеточное разбиение будем обозначать через $P(\Gamma)$.

ТЕорема 3. Существует такая функция $\varphi$, удовлетворяющая условию косой симметрии (3.8), что для любой области D на поверхности, которая состоит из конечного числа клеток и ограничена конечным числом простых попарно непересекаюшихся замкнутых ломаных, реализуюших симметрическую разность двух димерных покрытий графа Г, выполнено условие

$$
\psi(\partial D)=0 \bmod 2,
$$

где функиия $\psi$ определяется соотношением (3.16). 
ДокАЗАТЕЛЬСТво. Пусть $\varphi_{0}$ - функция, удовлетворяющая условию косой симметрии (3.8). Рассмотрим двумерную коцепь $c_{0}$, значение которой на любой клетке $\sigma$ определяется следующим образом. Так как наш граф Г лежит на ориентируемой поверхности $P$, то всякая клетка $\sigma$ приобретает ориентацию, согласованную с ориентацией поверхности, а вместе с ней ориентацию, т.е. направление обхода, приобретает и замкнутая ломаная $\partial \sigma$. Положим

$$
c_{0}(\sigma)=1+\varphi_{0}(\partial \sigma) \bmod 2 .
$$

Как всякая двумерная коцепь на поверхности, коцепь $c_{0}$ является коциклом. Докажем, что она является кограницей. Для этого достаточно проверить выполнение условия

$$
c_{0}(P)=0 \bmod 2 .
$$

Пусть $f, e$ и $v$ обозначают количество граней, ребер и вершин клеточного разбиения $P(\Gamma)$. Поскольку мы предполагаем, что граф $Г$ обладает димерным покрытием, число $v$ четно. Используя условие косой симметрии (3.8), подсчитаем $c_{0}(P)$ :

$$
c_{0}(P)=\sum_{\sigma \in P} c_{0}(\sigma)=\sum_{\sigma \in P}\left(1+\varphi_{0}(\partial \sigma)\right)=f+e=v+\chi=0 \bmod 2,
$$

так как эйлерова характеристика $\chi=2(1-g)$ поверхности $P$ четна. Тогда существует одномерная коцепь $x$, для которой выполнено равенство

$$
c_{0}=\partial^{*} x \text {. }
$$

Рассмотрим новую функцию

$$
\varphi(p, q)=\varphi_{0}(p, q)+x(p, q) \bmod 2,
$$

которая также удовлетворяет условию косой симметрии (3.8). При этом в силу соотношений $(4.2),(4.5)$ для любой двумерной клетки $\sigma$, ориентация которой согласована с ориентацией поверхности $P$, мы имеем

$$
1+\varphi(\partial \sigma)=0 \bmod 2 .
$$

По условию теоремы область $D$ на поверхности $P$ ограничена конечным числом простых непересекающихся ломаных $\gamma_{i}$. Натянем на каждую ломаную $\gamma_{i}$ свой вспомогательный диск $d_{i}$. Получим, вообще говоря, новую замкнутую поверхность $P^{\prime}$. Ориентацию каждого вспомогательного диска $d_{i}$ выбираем когерентно с ориентацией области $D$. Таким образом, если $\gamma_{i}(t), 0 \leqslant t \leqslant 1,-$ параметризованная ломаная, соответствующая положительно ориентированной компоненте края области $D$, то $\gamma_{i}(1-t), 0 \leqslant t \leqslant 1$, - та же ломаная с противоположным направлением обхода. Поскольку набор замкнутых ломаных $\gamma_{i}$ реализует симметрическую разность двух димерных покрытий, то число вершин, содержащихся в области $D$, четно и число ребер $l\left(\gamma_{i}\right)$ каждой ломаной $\gamma_{i}$ также четно. Используя равенство (4.4) для функции $\varphi$ и поверхности $P^{\prime}$, получаем

$$
\sum_{\sigma \in P^{\prime}}(1+\varphi(\partial \sigma))=0 \bmod 2
$$


В силу равенства (4.7) ненулевой вклад в левую часть равенства (4.8) дают лишь диски $d_{i}$. Из условия косой симметрии (3.8) для функции $\varphi$ и четности числа ребер $l\left(\gamma_{i}\right)$ ломаной $\gamma_{i}$ мы получаем

$$
1+\varphi\left(\partial d_{i}\right)=1+\varphi\left(\gamma_{i}(1-t)\right)=1+l\left(\gamma_{i}\right)+\varphi\left(\gamma_{i}(t)\right)=1+\varphi\left(\gamma_{i}(t)\right) \bmod 2 .
$$

Подставляя соотношения (4.7), (4.9) в левую часть равенства (4.8), получаем равенство (4.1). Теорема доказана.

Симметрическая разность двух димерных покрытий $U_{0}$ и $U_{i}, i=1,2$, представляет собой конечное множество простых замкнутых непересекающихся ломаных $\left\{\gamma_{1}^{i}, \gamma_{2}^{i}, \ldots\right\}$, причем каждая компонента связности из $U_{0} \Delta U_{1}$ состоит из попеременно чередующихся ребер из димерных покрытий $U_{0}$ и $U_{1}$ соответственно. Аналогично, $U_{0} \Delta U_{2}$ состоит из попеременно чередующихся ребер из димерных покрытий $U_{0}$ и $U_{2}$ соответственно.

Лемма 1. Имеет место равенство

$$
\left(U_{0} \Delta U_{1}\right) \Delta\left(U_{0} \Delta U_{2}\right)=U_{1} \Delta U_{2} .
$$

ДокАЗАТЕЛЬСтво. Пусть ребро $е$ принадлежит левой части равенства (4.10), и предположим, что $e \in U_{0} \Delta U_{1}$, но $e \notin U_{0} \Delta U_{2}$. Тогда $e \in\left(U_{0} \cup U_{1}\right) \backslash\left(U_{0} \cap U_{1}\right)$. Если $e \in U_{0}$, то $e \notin U_{1}$ и поэтому $e \in U_{2}$. Отсюда $e \in U_{1} \Delta U_{2}$. Если же $e \in U_{1}$, то $e \notin U_{0}$. Поэтому $e \notin U_{2}$. Отсюда $e \in U_{1} \Delta U_{2}$. Совершенно аналогично рассматривается случай $e \in U_{0} \Delta U_{2}$, но $e \notin U_{0} \Delta U_{1}$.

Пусть ребро $e$ принадлежит правой части равенства (4.10), и предположим, что $e \in U_{1}$ и $e \notin U_{2}$. Если $e \notin U_{0}$, то $e \in U_{0} \Delta U_{1}$ и $e \notin U_{0} \Delta U_{2}$. Значит, $e$ принадлежит левой части равенства (4.10). Если $e \in U_{0}$, то $e \notin U_{0} \Delta U_{1}$ и $e \in U_{0} \Delta U_{2}$. Значит, $е$ принадлежит левой части равенства (4.10). Совершенно аналогично рассматривается случай $e \in U_{2}$ и $e \notin U_{1}$. Лемма доказана.

Следуюшая теорема является непосредственным следствием соотношения (3.15).

Теорема 4. Для любых трех димерных покрытий $U_{0}, U_{1}, U_{2}$ и любой функиии $\varphi$, удовлетворяющей условию косой симметрии (3.8), имеет место следуюшее соотношение

$$
\psi\left(U_{0} \Delta U_{1}\right)+\psi\left(U_{0} \Delta U_{2}\right)=\psi\left(U_{1} \Delta U_{2}\right) \bmod 2,
$$

где функиия $\psi$ определяется равенством (3.16).

Пусть теперь граф Г представляет собой достаточно мелкое клеточное разбиение ориентируемой замкнутой поверхности $P$. А именно, пусть ломаные $a_{i}^{0}$, $b_{i}^{0}, 1 \leqslant i \leqslant g$, составленные из ребер графа $\Gamma$, являются системой образующих группы $H_{1}\left(P(\Gamma), \mathbb{Z}_{2}\right)$ и ломаные $a_{i}^{0}, b_{i}^{0}, a_{i}^{0}+b_{i}^{0}$ для различных индексов $i$ не пересекаются. Следуя процедуре $\S 2$, по графу $Г$ построим новый граф $\operatorname{cl}(\Gamma)$. При этом каждая вершина графа $\Gamma$, соединенная с $2 k, k>1$, другими вершинами, заменяется на $6(k-1)$ новых вершин. $\mathrm{K}$ ребрам графа $\Gamma$ в графе $\mathrm{cl}(\Gamma)$ добавляются новые ребра, соединяющие только вершины, возникшие из одной и той же вершины графа Г. Если граф Г удовлетворяет условию $(Г)$, то легко проверить, что новый граф также удовлетворяет этому условию.

Каждому циклу $a_{i}^{0}, b_{i}^{0}, a_{i}^{0}+b_{i}^{0}, 1 \leqslant i \leqslant g$, из группы $Z_{1}\left(\Gamma, \mathbb{Z}_{2}\right)$ сопоставим в соответствии с процедурой $\S 2$ следующие димерные покрытия графа $\mathrm{cl}(\Gamma)$ : 
$U_{1 i}=U\left(a_{i}^{0}\right), U_{2 i}=U\left(b_{i}^{0}\right)$ и $U_{3 i}=U\left(a_{i}^{0}+b_{i}^{0}\right), 1 \leqslant i \leqslant g$. В качестве димерного покрытия $U_{0}$ выберем димерное покрытие графа $\mathrm{cl}(\Gamma)$, соответствующее циклу, который равен нулю на любом ребре графа $Г$. Тогда димерное покрытие $U_{0}$ содержит только ребра, соединяющие вершины, возникшие из одной и той же вершины графа $Г$. Поэтому симметрические разности димерных покрытий

$$
a_{i}=U_{0} \Delta U_{1 i}, \quad b_{i}=U_{0} \Delta U_{2 i}, \quad U_{0} \Delta U_{3 i}
$$

представляют собой ломаные, которые содержат ребра ломаных $a_{i}^{0}, b_{i}^{0}, a_{i}^{0}+b_{i}^{0}$ соответственно, а также ребра, соединяюшие вершины, возникшие из одной и той же вершины графа $Г$. Поэтому если ломаные $a_{i}^{0}, b_{i}^{0}, a_{i}^{0}+b_{i}^{0}$ на графе $\Gamma$ не пересекаются для различных индексов $i$, то ломаные $a_{i}, b_{i}, U_{0} \Delta U_{3 i}$ на графе $\operatorname{cl}(\Gamma)$ также не пересекаются для различных индексов $i$, т.е.

$$
\left(U_{0} \Delta U_{k i}\right) \cap\left(U_{0} \Delta U_{l j}\right)=\varnothing, \quad 1 \leqslant i<j \leqslant g, k, l=\{1,2,3\} .
$$

Нетрудно видеть, что ломаная $U_{0} \Delta U_{3 i}$ гомологична ломаной $a_{i}+b_{i}$. В самом деле, симметрическая разность этих двух замкнутых ломаных $U_{0} \Delta U_{3 i}$ и $a_{i}+b_{i}$ представляет собой набор замкнутых ломаных, составленных из новых ребер, соединяющих вершины, возникшие из одной и той же вершины графа Г. Такими замкнутыми ломаными могут быть лишь треугольники. Все они гомологичны нулю.

Зададим деформацию разбиения $P(\operatorname{cl}(\Gamma))$, стягивая в точки все новые ребра, соединяющие только вершины, возникшие из одной и той же вершины графа $\Gamma$. Ясно, что группа гомологий $H_{1}\left(P(\operatorname{cl}(\Gamma)), \mathbb{Z}_{2}\right)$ изоморфно отображается на $H_{1}\left(P(\Gamma), \mathbb{Z}_{2}\right)$. Поэтому, поскольку ломаные $a_{i}^{0}, b_{i}^{0}, 1 \leqslant i \leqslant g$, составленные из ребер графа $\Gamma$, являются системой образующих группы $H_{1}\left(P(\Gamma), \mathbb{Z}_{2}\right)$, ломаные $a_{i}, b_{i}, 1 \leqslant i \leqslant g$, составленные из ребер графа $\mathrm{cl}(\Gamma)$, также являются системой образующих группы $H_{1}\left(P(\operatorname{cl}(\Gamma)), \mathbb{Z}_{2}\right)$. Одномерная группа гомологий $H_{1}\left(P(\operatorname{cl}(\Gamma)), \mathbb{Z}_{2}\right)$ разлагается в прямую сумму своих подгрупп:

$$
H_{1}\left(P(\operatorname{cl}(\Gamma)), \mathbb{Z}_{2}\right)=\bigoplus_{i=1}^{g} \mathbb{Z}_{2}\left\{a_{i}, b_{i}\right\},
$$

где $\mathbb{Z}_{2}\left\{a_{i}, b_{i}\right\}$ — подгруппа, аддитивно порожденная образующими $a_{i}, b_{i}$ с коэффициентами из группы $\mathbb{Z}_{2}$, т. е. подгруппа, состоящая из четырех элементов: $0, a_{i}, b_{i}$ и $a_{i}+b_{i}$.

Рассмотрим множество $U(\operatorname{cl}(\Gamma))$ всех димерных покрытий графа $\mathrm{cl}(\Gamma)$. Для любых двух димерных покрытий их симметрическая разность в соответствии с равенством (3.4) представляется в виде несвязного объединения конечного числа замкнутых ломаных. При фиксированном димерном покрытии $U_{0} \in U(\operatorname{cl}(\Gamma))$ функция $\psi$, построенная в теореме 3 , определяет функцию $\psi\left(U_{0} \Delta U\right)$ на множестве $U(\operatorname{cl}(\Gamma))$. В силу равенства (3.4) симметрическая разность $U_{0} \Delta U$ реализует некоторый одномерный класс гомологий $\left[U_{0} \Delta U\right] \in H_{1}\left(P(\operatorname{cl}(\Gamma)), \mathbb{Z}_{2}\right)$. Если для двух димерных покрытий $U_{1}, U_{2} \in U(\operatorname{cl}(\Gamma))$ симметрические разности $U_{0} \Delta U_{1}$ и $U_{0} \Delta U_{2}$ реализуют один класс гомологий из группы $H_{1}\left(P(\operatorname{cl}(\Gamma)), \mathbb{Z}_{2}\right)$, то их симметрическая разность, которая в силу леммы 1 совпадает с симметрической разностью $U_{1} \Delta U_{2}$, является границей некоторой ориентируемой области, возможно несвязной, на поверхности $P$. Поэтому из теорем 3 и 4 мы получаем

$$
\psi\left(U_{0} \Delta U_{1}\right)+\psi\left(U_{0} \Delta U_{2}\right)=\psi\left(U_{1} \Delta U_{2}\right)=0 \bmod 2,
$$


т. е. значения $\psi\left(U_{0} \Delta U_{1}\right)$ и $\psi\left(U_{0} \Delta U_{2}\right)$ совпадают по модулю 2. Таким образом $\mathbb{Z}_{2}$-значная функция $\psi\left(U_{0} \Delta U\right)$ на множестве димерных покрытий $U(\operatorname{cl}(\Gamma))$ графа $\operatorname{cl}(\Gamma)$ порождает $\mathbb{Z}_{2}$-значную функцию на подмножестве $\left\{\left[U_{0} \Delta U\right] \mid U \in\right.$ $U(\operatorname{cl}(\Gamma))\}$ группы одномерных гомологий $H_{1}\left(P(\operatorname{cl}(\Gamma)), \mathbb{Z}_{2}\right)$.

Докажем, что множество $\left\{\left[U_{0} \Delta U\right] \mid U \in U(\operatorname{cl}(\Gamma))\right\}$ совпадает со всей группой гомологий $H_{1}\left(P(\mathrm{cl}(\Gamma)), \mathbb{Z}_{2}\right)$, т. е. любой элемент группы (4.14) реализуется в виде симметрической разности димерного покрытия $U_{0}$ и другого димерного покрытия графа $\mathrm{cl}(\Gamma)$. В силу определений (4.12) этим свойством обладают элементы $a_{i}, b_{i}, 1 \leqslant i \leqslant g$. Симметрическая разность $U_{0} \Delta U_{3 i}$ гомологична ломаной $a_{i}+b_{i}$, т. е. она реализует класс гомологий $\left[a_{i}+b_{i}\right]$. Докажем, что несвязная ломаная $a_{i}+a_{j}$ может быть реализована в виде симметрической разности димерного покрытия $U_{0}$ и другого димерного покрытия графа $\mathrm{cl}(\Gamma)$. Рассмотрим димерное покрытие $U_{0} \Delta\left(\left(U_{0} \Delta U_{1 i}\right) \Delta\left(U_{0} \Delta U_{1 j}\right)\right)$ графа $\operatorname{cl}(\Gamma)$. Тогда симметрическая разность димерного покрытия $U_{0}$ и этого димерного покрытия равна $\left(U_{0} \Delta U_{1 i}\right) \Delta\left(U_{0} \Delta U_{1 j}\right)$ и совпадает с $a_{i}+a_{j}$ в силу соотношений (4.12) и (4.13). Совершенно аналогично можно доказать, что любой элемент группы (4.14) peализуется в виде симметрической разности димерного покрытия $U_{0}$ и другого димерного покрытия графа $\operatorname{cl}(\Gamma)$.

Значение функции $\psi$ на элементе группы (4.14) мы определим как значение функции $\psi$ на соответствующей симметрической разности димерного покрытия $U_{0}$ и другого димерного покрытия графа $\operatorname{cl}(\Gamma)$. Пусть $\gamma_{i} \in \mathbb{Z}_{2}\left\{a_{i}, b_{i}\right\}, 1 \leqslant$ $i \leqslant g$. Тогда из соотношения (3.16) и соотношений (4.13) следует, что

$$
\psi\left(\gamma_{i}\right)+\psi\left(\gamma_{j}\right)+\psi\left(\gamma_{i}+\gamma_{j}\right)=0 \bmod 2
$$

для $i \neq j$. Таким образом, для элементов различных групп $\mathbb{Z}_{2}\left\{a_{i}, b_{i}\right\}$ выполнено соотношение линейности (4.15). Для элементов одной группы $\mathbb{Z}_{2}\left\{a_{i}, b_{i}\right\}$ возможны два варианта

$$
\begin{aligned}
& \psi\left(a_{i}\right)+\psi\left(b_{i}\right)+\psi\left(a_{i}+b_{i}\right)=0 \bmod 2, \\
& \psi\left(a_{i}\right)+\psi\left(b_{i}\right)+\psi\left(a_{i}+b_{i}\right)=1 \bmod 2 .
\end{aligned}
$$

Предположим, что к каждой вершине графа $Г$ подходит ровно четыре ребра и циклы $a_{i}^{0}, b_{i}^{0}, 1 \leqslant i \leqslant g$, составляющие систему образуюших группы $H_{1}\left(P(\Gamma), \mathbb{Z}_{2}\right)$, для различных индексов $i$ не пересекаются, а для одинаковых индексов $i$ пересекаются в одной вершине графа Г трансверсально, т. е. любые два соседних ребра, между которыми нет других ребер, принадлежат различным циклам. Докажем, что в этом случае выполнено соотношение (4.17), где $a_{i}$ и $b_{i}$ определяются как разности димерных покрытий (4.12) графа $\mathrm{cl}(\Gamma)$, а число $\psi\left(a_{i}+b_{i}\right)$ определяется как значение функции $\psi$ на третьей симметрической разности $U_{0} \Delta U_{3 i}$, входящей в определение (4.12). В самом деле, в силу равенства (4.11) справедливо следующее равенство по модулю $2: \psi\left(a_{i}\right)+\psi\left(b_{i}\right)=\psi\left(U_{1 i} \Delta U_{2 i}\right)$. Симметрические разности $U_{0} \Delta U_{3 i}$ и $U_{1 i} \Delta U_{2 i}$ представляют собой две замкнутые ломаные, которые совпадают всюду, за исключением семи ребер, соединяющих точки, отвечающие вершине пересечения ломаных $a_{i}^{0}$ и $b_{i}^{0}$ на графе Г. Эти семь ребер образуют два треугольника, соединенные ребром. Ломаная $U_{0} \Delta U_{3 i}$ проходит ровно через одно ребро каждого из двух треугольников, а ломаная $U_{1 i} \Delta U_{2 i}-$ через два дополнительных ребра каждого из двух треугольников. Выберем на 
ломаных $U_{0} \Delta U_{3 i}$ и $U_{1 i} \Delta U_{2 i}$ направления, совпадающие на общих частях этих ломаных. Тогда направленная ломаная $U_{0} \Delta U_{3 i}$ индуцирует на двух треугольниках противоположные ориентации. Направленная ломаная $U_{1 i} \Delta U_{2 i}$ индуцирует на тех же треугольниках ориентации, противоположные тем, которые индуцирует направленная ломаная $U_{0} \Delta U_{3 i}$. В силу условия косой симметрии $(3.8)$, не меняя значения функции $\psi$ на ломаной, можно изменить ориентацию на двух паpax ребер треугольников. Тогда левая часть равенства (4.17) в этом случае равна сумме значений функции ориентации $\varphi$ на двух противоположно ориентированных треугольниках. Последнее выражение равно 1, поскольку по построению (см. равенство (4.7)) функция ориентации $\varphi$ равна 1 на треугольнике, ориентация которого согласована с ориентацией поверхности $P$, и в силу условия косой симметрии (3.8) она равна нулю на треугольнике, ориентация которого противоположна ориентации поверхности $P$.

Таким образом, геометрически естественным является лишь случай равенства (4.17). Для обшности рассмотрения мы будем учитывать оба равенства (4.16) и (4.17). Изменяя нумерацию в прямой сумме (4.14), мы можем считать, что для индексов $1 \leqslant i \leqslant g_{1}$ выполнено соотношение (4.17), а для индексов $g_{1}<i \leqslant$ $g$ - соотношение (4.16). Конечно, мы не исключаем возможности, когда для всех индексов $1 \leqslant i \leqslant g$ выполнено какое-либо одно из соотношений (4.16) и (4.17). Обозначим через $a_{i}^{*}$ и $b_{i}^{*}$ коциклы, реализующие классы когомологий в группе $H^{1}\left(P(\operatorname{cl}(\Gamma)), \mathbb{Z}_{2}\right)$, двойственные элементам $a_{i}, b_{i}$, т. е. $a_{i}^{*}\left(a_{j}\right)=\delta_{i j}$, $a_{i}^{*}\left(b_{j}\right)=0$ и $b_{i}^{*}\left(a_{j}\right)=0, b_{i}^{*}\left(b_{j}\right)=\delta_{i j}$. Тогда пользуясь равенствами (4.15), (4.16) и (4.17), можно проверить следующее тождество для любого димерного покрытия $U$ графа $\mathrm{cl}(\Gamma)$ :

$$
\begin{aligned}
& \begin{array}{c}
(-1)^{\psi\left(U_{0} \Delta U\right)}=\left(\prod _ { i = 1 } ^ { g _ { 1 } } \left[(-1)^{\left(1+\psi\left(a_{i}\right)\right) a_{i}^{*}\left(U_{0} \Delta U\right)+\left(1+\psi\left(b_{i}\right)\right) b_{i}^{*}\left(U_{0} \Delta U\right)}\right.\right. \\
\times \frac{1}{2}\left((-1)^{a_{i}^{*}\left(U_{0} \Delta U\right)+b_{i}^{*}\left(U_{0} \Delta U\right)}\right.
\end{array} \\
& \left.\left.\left.+(-1)^{a_{i}^{*}\left(U_{0} \Delta U\right)}+(-1)^{b_{i}^{*}\left(U_{0} \Delta U\right)}-1\right)\right]\right) \\
& \times \prod_{i=g_{1}+1}^{g}(-1)^{\psi\left(a_{i}\right) a_{i}^{*}\left(U_{0} \Delta U\right)+\psi\left(b_{i}\right) b_{i}^{*}\left(U_{0} \Delta U\right)} .
\end{aligned}
$$

Произведение (4.18) можно представить в виде суммы

$$
(-1)^{\psi\left(U_{0} \Delta U\right)}=\sum_{i=1}^{2^{2 g_{1}}} 2^{-g_{1}}(-1)^{n\left(x_{i}\right)}(-1)^{x_{i}\left(U_{0} \Delta U\right)+y[\varphi]\left(U_{0} \Delta U\right)},
$$

где коциклы $x_{i}$ выбираются по одному из каждого класса когомологий в группе $H^{1}\left(P(\mathrm{cl}(\Gamma)), \mathbb{Z}_{2}\right)$, который тождественно равен нулю на подгруппах $\mathbb{Z}_{2}\left\{a_{j}, b_{j}\right\}$ для всех индексов $g_{1}<j \leqslant g$. Число

$$
n(x)=\sum_{i=1}^{g_{1}}\left(1+x\left(a_{i}\right)+x\left(b_{i}\right)+x\left(a_{i}\right) x\left(b_{i}\right)\right)
$$

совпадает по модулю 2 с числом подгрупп $\mathbb{Z}_{2}\left\{a_{j}, b_{j}\right\}$ с индексами $1 \leqslant j \leqslant g_{1}$, на которых коцикл $x$ тождественно равен нулю. Коцикл $y[\varphi]$ определяется функ- 
цией $\varphi$

$$
y[\varphi]=\sum_{i=1}^{g_{1}}\left(\left(1+\psi\left(a_{i}\right)\right) a_{i}^{*}+\left(1+\psi\left(b_{i}\right)\right) b_{i}^{*}\right)+\sum_{i=g_{1}+1}^{g}\left(\psi\left(a_{i}\right) a_{i}^{*}+\psi\left(b_{i}\right) b_{i}^{*}\right),
$$

где функция $\psi$ задается соотношением (3.16).

Для функции $\varphi$, построенной в теореме 3 , положим

$$
\varphi\left[x_{i}\right](p, q)=\varphi(p, q)+x_{i}(p, q)+y[\varphi](p, q) \bmod 2 .
$$

Поскольку $x_{i}$ и $y[\varphi]$ - одномерные коцепи на графе $\operatorname{cl}(\Gamma)$ со значениями в группе $\mathbb{Z}_{2}$, то функция $\varphi\left[x_{i}\right]$ удовлетворяет соотношению косой симметрии $(3.8)$, как и функция $\varphi$. Пусть $A\left[x_{i}\right]$ обозначает матрицу, которая определяется формулами $(2.10),(3.7)$, где функцию $\varphi$ нужно заменить функцией $\varphi\left[x_{i}\right]$, определенной соотношением (4.22). Аналогично с помощью равенства (3.6) задается функция $\tau\left[x_{i}\right](U)$ на множестве димерных покрытий графа $\operatorname{cl}(\Gamma)$.

Докажем, что производящую функцию (2.8) димерных покрытий графа $\mathrm{cl}(\Gamma)$ можно представить в виде следующей линейной комбинации $2^{2 g_{1}}$ пфаффианов структурных матриц $A\left[x_{i}\right]$ :

$$
Z=\sum_{i=1}^{2^{2 g_{1}}} 2^{-g_{1}}(-1)^{n\left(x_{i}\right)+\tau\left[x_{i}\right]\left(U_{0}\right)} \operatorname{Pf}\left(A\left[x_{i}\right]\right) .
$$

Действительно, пользуясь формулами (2.16), (3.5), (3.15), (3.16), (4.19) и (4.22), сумму (4.23) можно преобразовать следующим образом:

$$
\begin{aligned}
Z & =\sum_{i=1}^{2^{2 g_{1}}} 2^{-g_{1}}(-1)^{n\left(x_{i}\right)+\tau\left[x_{i}\right]\left(U_{0}\right)} \sum_{U}(-1)^{\tau\left[x_{i}\right](U)} z^{U} \\
& =\sum_{i=1}^{2^{2 g_{1}}} 2^{-g_{1}}(-1)^{n\left(x_{i}\right)} \sum_{U}(-1)^{\psi\left(U_{0} \Delta U\right)+x_{i}\left(U_{0} \Delta U\right)+y[\varphi]\left(U_{0} \Delta U\right)} z^{U}=\sum_{U} z^{U} .
\end{aligned}
$$

На первый взгляд формула (4.23) зависит от выбора представителей $x_{i}$ классов когомологий из $H^{1}\left(P(\operatorname{cl}(\Gamma)), \mathbb{Z}_{2}\right)$. Докажем, что это не так. Из определения (3.6) следует, что при добавлении к ориентации $\varphi$ одномерной кограницы $\partial^{*} z \mathrm{c}$ коэффициентами в группе $\mathbb{Z}_{2}$ мы получаем для любого димерного покрытия $U$

$$
\tau\left[\varphi+\partial^{*} z\right](U)=\tau[\varphi](U)+\sum_{i=1}^{M / 2}\left(z\left(p_{i}\right)+z\left(q_{i}\right)\right) \bmod 2 .
$$

В силу определения димерного покрытия сумма в правой части равенства (4.25) является суммой по всем вершинам графа $\mathrm{cl}(\Gamma)$. Подставляя соотношение (4.25) в определение $(2.16),(3.5),(3.6)$ пфаффиана структурной матрищы $A[\varphi]$, мы получаем соотношение

$$
\operatorname{Pf}\left(A\left[\varphi+\partial^{*} z\right]\right)=(-1)^{\sum_{p \in V} z(p)} \operatorname{Pf}(A[\varphi]) .
$$

Из соотношений (4.25), (4.26) следует независимость формулы (4.23) от выбора представителей $x_{i}$ классов когомологий из $H^{1}\left(P(\operatorname{cl}(\Gamma)), \mathbb{Z}_{2}\right)$. 
В силу равенства (4.25) для ориентации $\varphi\left[x_{i}\right]$ и димерного покрытия $U_{0}$ представители $x_{i}$ классов когомологий из $H^{1}\left(P(\operatorname{cl}(\Gamma)), \mathbb{Z}_{2}\right)$ можно выбрать так, что $\tau\left[x_{i}\right]\left(U_{0}\right)=0 \bmod 2$, т. е. так, что формула (4.23) упрощается. После этого представители $x_{i}$ классов когомологий в формуле (4.23) можно изменять лишь на кограницы $\partial^{*} z_{i}$ тех нульмерных коцепей $z_{i}$, которые удовлетворяют условию

$$
\sum_{p \in V} z_{i}(p)=0 \bmod 2
$$

ЗАКЛЮЧИТЕЛЬНЫЕ ЗАМЕЧАНИЯ. 1. Сам факт существования разложения производящей функции $Z$ димерных покрытий графа $\mathrm{cl}(\Gamma)$ в виде линейной комбинации не более чем $2^{2 g}$ пфаффианов структурных матриц $A\left[x_{i}\right]$ вытекает из теоремы Стоуна-Вейерштрасса, из полноты системы функций $(-1)^{x_{i}(\gamma)}$, $\gamma \in H_{1}\left(P(\operatorname{cl}(\Gamma)), \mathbb{Z}_{2}\right)$, и из того, что функция $\psi$ корректно определена на группе $H_{1}\left(P(\operatorname{cl}(\Gamma)), \mathbb{Z}_{2}\right)$.

2. Разложение (4.23) дает объяснения известных формул, представляющих статистическую сумму в виде линейной комбинации пфаффианов структурных матриц. Так, в случае квадратной решетки со свободными граничными условиями граф можно расположить на сфере. Это соответствует тому, что разложение (4.23) состоит из одного слагаемого. Аналогично, в случае периодического граничного условия в одном измерении граф можно расположить на сфере, т. е. разложение (4.23) тоже состоит из одного слагаемого. Наконец, в случае двоякопериодических граничных условий граф располагается на двумерном торе. Следовательно, разложение (4.23) состоит не более чем из четырех слагаемых.

\section{ЛитеРАтУРА}

1. McCoy B. M., Tai Tsun Wu. The Two-dimensional Ising Model. Harvard Univ. Press, Cambridge, Mass. (1973).

2. van der Waerden B. L. Lange Reichweite der regelmässigen Atomanordnung in Mischkristallen. Z. Physik, 118, 473-488 (1941).

3. Fisher M. E. On the Dimer Solution of Planar Ising Models. J. Math. Phys., 7, No. 10, 1776-1781 (1966).

4. Бурбаки Н. Алгебра. Модули, кольца, формы. Наука, М. (1966).

Математический институт

им. В. А. Стеклова
Поступило в редакцию 22 февраля 1995 г. 